\title{
INERTIAL SENSING IN A HAND HELD DYNAMOMETER
}

\author{
Peter H. Veltink ${ }^{1}$, Daniël M. Nieuwland ${ }^{1}$, Jaap Harlaar ${ }^{2}$ and Chris T.M. Baten ${ }^{3}$ \\ ${ }^{1}$ University of Twente, P.O. Box 217, 7500 AE Enschede, the Netherlands \\ ${ }^{2}$ Dept. Rehabilitation, VU Hospital, Amsterdam, the Netherlands \\ ${ }^{3}$ Roessingh Research and Development, Enschede, the Netherlands \\ e-mail:P.H.Veltink@el.utwente.nl
}

\begin{abstract}
Two methods for kinematic sensing in a handheld dynamometer using accelerometers and gyroscopes are presented. The first method integrates the angular velocity signal from the gyroscope, after calibration of gyroscope offset and joint angle from a static period immediately preceeding each measurement. The second method estimates tangential and radial accelerations, enabling the estimation of the gravity components in the accelerometer signals under dynamic conditions, and thus angle reconstruction. The second method appeared to perform best in preliminary tests.
\end{abstract}

\section{INTRODUCTION}

In physical therapy practice muscle function is commonly investigated during physical examination. This assessment is subjectively performed by moving a limb segment and assessing the resistance against such a movement. Harlaar et al [1] developed a hand-held dynamometer, called CAHNDY, which enables to perform this assessement in an objective manner. The system consists of a force transducer mounted in the handle with which the therapist moves a leg or arm segment and a flexible goniometer mounted at the joint.

The objective of the current study is to integrate both the force transducer and the kinematic sensing in the handle, with which the leg is moved, thus simplifying the measurement. Kinematic sensing is done using accelerometers and gyroscopes, which are both inertial sensor. The sensors should deliver an accurate estimate of segment angle and angular velocity, which can be real-time used in the dynamometer measurements.

\section{INERTIAL SENSING}

The use of accelerometers in human movement analysis has been investigated by several authors [2-4]. A uniaxial accelerometer measures one component of the equivalent acceleration, which is the sum of the actual acceleration $\bar{a}$ and the gravitational acceleration:

$$
a_{e q}=(\vec{a}-\vec{g}) \cdot \vec{u}
$$

The direction of the sensitive axis of the sensor is indicated by the unit vector $\vec{u}$.

Generally, during movements, actual accelerations can not be distinguished from changing gravitational acceleration components when the sensor is simultanously accelerating and rotating.
Additional useful kinematic information can be obtained using uni-axial rate gyroscopes [5], which measure one component of the angular velocity $\vec{\omega}$.

\section{Inertial sensing methods}

\section{METHODS}

We propose to combine accelerometers and gyroscopes for improved kinematic sensing and compared two methods for assessing shank angle during movements of the shank in a sitting position of a subject. We assume movements limited to the sagittal plane, and use tangential and radial accelerometers in the sagittal plane at a position along the shank, and a uniaxial gyroscope measuring angular velocities in the sagittal plane (fig. 1).

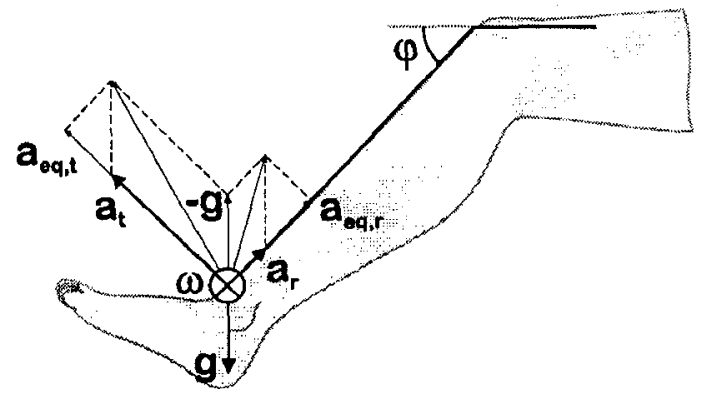

Fig. 1. Assessing shank angle and angular velocity using tangential and radial accelerometers and a uni-axial gyroscope measuring in the sagittal plane.

Method $I$ requires a static period at the start of the test. In this period the accelerometers provide an estimate of the shank angle with respect to gravity (equation (I) with $\vec{a}=\overrightarrow{0}$ ) and the offset of the gyroscope can be determined. The joint angle is estimated by integrating the gyroscope-signal after offset-correction for drift.

Method 2 makes use of the assumption that the knee joint is not linearly accelerating during the rotation of the shank. Under this condition the shank can be considered as a pendulum, and the tangential and radial components of the actual accelerations measured at a position along the shank can be estimated using the signal of the gyroscope located at the same position, enabling the estimation of knee angle by:

$$
\varphi=\tan ^{-1}\left(\frac{a_{e q, r}-r \omega^{2}}{a_{e q, t}-r \dot{\omega}}\right)
$$


$\dot{\omega}$ is obtained by differentiating the gyroscope signal $\omega$; the distance from knee axis to measurement position $r$ has to be measured seperately.

\section{Experimental methods}

In order to examplify the performance of both methods an experimental test was carried out in a healthy subject, sitting on a table, his shank free to move. The tangential and radial accelerometers (ICsensors ${ }^{\circledR}, 5 \mathrm{~g}$ ) and a uni-axial gyroscope (Murata ${ }^{\circledR}$ ) were attached to his shank at an estimated distance of $38 \mathrm{~cm}$ from the axis of the knee. The subject performed cyclical movements with his shank, starting at a static angle. For comparison, a Penny \& Giles ${ }^{\circledR}$ 2-axial flexible knee goniometer was used to obtain an independent measurement of knee angle. The signals were sampled in a 486 PC system at $100 \mathrm{~Hz}$, after 4th order presampling filtering at a cut-off frequency of $35 \mathrm{~Hz}$, and subsequently digitally filtered using a 4 th order Butterworth filter with a cut-off frequency of $5 \mathrm{~Hz}$.

\section{RESULTS}

In method 1 the determination of the gyroscope offset in a static period of 2 seconds immediately preceeding the measurement, markedly decreased drift (figure 2b). When the gyroscope offset was determined several minutes earlier a drift of $285 \mathrm{deg}$ in $10 \mathrm{~s}$ occured. However, the figure indicates that during short measurements of $10 \mathrm{~s}$ the gyroscope offset can change such that drift reappears (in the example after $8 \mathrm{~s}$ ). (in this example RMS difference with respect to goniometer was $13.3 \mathrm{deg}$ ). Method 2 clearly performed best, yielding a reconstructed knee angle, very close to the angle measured with the goniometer (figure 2b): the RMS difference in this example was $4.4 \mathrm{deg}$.

\section{DISCUSSION}

Method 2 clearly performed best in our preliminary measurements, avoiding drift, which may occur with integration in method 1. Method 2 requires a known distance $r$ between knee axis and sensor system, which needs to be measured anyway for estimating knee torque from the force measured in the handle. The method assumes a fixed knee axis, which is not actually true. However, a sensitivity analysis showed that the method is relatively insensitive for errors in the distance $r$. A further requirement is that the joint axis and the other segment (thigh) should not move or rotate, which in many practical applications can be realised. The remaining angle difference between the reconstruction from inertial sensors and goniometer may partly be due to the inaccuracy of the goniometer. A comparison with a more accurate reference (e.g. optokinetic system) still has to be performed.

\section{CONCLUSION}

The preliminary results suggest that method 2 performs best, and is therefore suggested for use in the hand held dynamometer. Method 1 appears relatively unreliable, because small gyroscope offset changes introduce large drifts even in short measurements.

\section{REFERENCES}

[1] J. Harlaar, M.E. Roebroeck and G.J. Lankhorst, "The Computer Assisted Handheld Dynamometer, a Low Cost Instrument for Muscle Function Assessment in Rehabilitation Medicine", Med. \& Biol. Eng. \& Comput., 1996, accepted.

[2] J.R.W. Morris, "Accelerometry - a Technique for the Measurement of Human Body Movements", J. Biomech., vol. 6, 1973, pp. 729-736.

[3] A.Th.M. Willemsen, J.A. van Alsté and H.B.K. Boom, "Real-time Gait Assessment Utilizing a New Way of Accelerometry", J. Biomech., Vol. 23, 1990, pp. 859863.

[4] Peter H. Veltink and Rob C. van Lummel (eds.), Dynamic Analysis using Body Fixed Sensors,.2nd World Congress of Biomechanics, 1994 (ISBN 90-9007328-0).

[5] J. Söderkvist, "Micromachined gyroscopes", Sensors and Actuators A, vol. 43, 1994, pp. 65-71.

a.

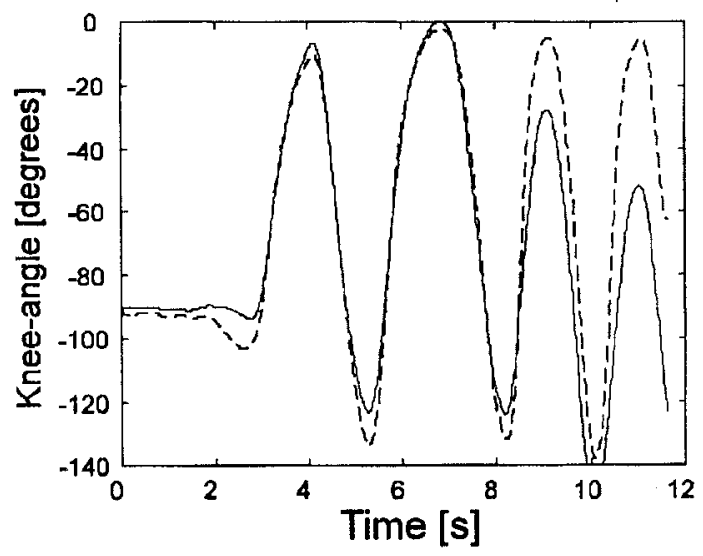

b.

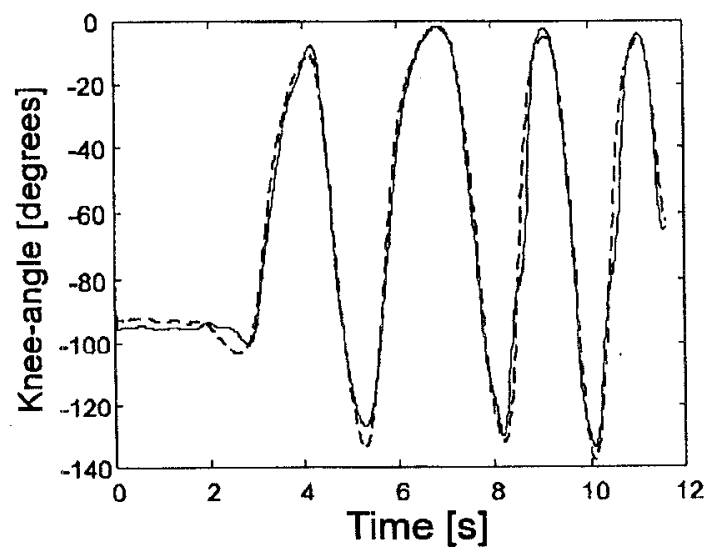

Fig. 2. Knee angle reconstruction from accelerometers and gyroscopes (solid lines) using both methods (a: method 1; b: method 2) The dashed line gives the goniometer measurement. 